\title{
THE MEASUREMENT AND SIGNIFICANCE OF GENOTYPE-ENVIRONMENT INTERACTIONS IN GRASSES
}

\author{
E. L. BREESE \\ Welsh Plant Breeding Station, Aberystwyth
}

Received 20.ii.68

\section{INTRODUCTION}

THE occurrence of genotype-environment interactions has long provided a major challenge in obtaining a fuller understanding of the genetic control of variability. They have posed serious problems in interpreting evolutionary trends and have hampered the rationalisation of policy and procedure in breeding for improved performance in economic crops.

Until recently these interactions have not proved tractable in biometrical analysis, but developments in two independent investigations lend hope that many of the difficulties may finally be overcome. At the Waite Institute, Finlay and Wilkinson (1963) have developed a statistical technique to compare the yield performance of a set of cereal varieties grown at several centres for several seasons. This involves computing for each variety the regression of individual yield on the mean yield of all varieties for each site and season. For the varieties and sites tested these regressions had a high degree of linearity and have been used as measures of the adaptabilities of the varieties. It is interesting to note that a similar technique yielding similar results was reported by Yates and Cochran (1938), but was apparently not further developed or used.

More recently, at the University of Birmingham, the genetical component analyses proposed by Mather (1949) have been developed to take into account effects due to genotype-environment interactions (Mather and Morley Jones, 1958; Jinks and Stevens, 1959). This involves partitioning quantitative data according to genetic and environmental effects and the interaction of these. The method has been adapted to the analysis of data from inbred lines and their crosses in Nicotiana rustica (Bucio Alanis, 1966; Bucio Alanis and Hill, 1966; Perkins and Jinks, 1968), where it has been found that the degree of interaction can be expressed as a linear function of the effect of the environment.

The significant feature in each of these different investigations was the discovery that genetic expression could be simply and predictably related to the environment when the latter was measured by its effect on the character under study. This paper discusses the results obtained when these methods are applied to yield data in herbage plants, which perhaps of all cultivated crops have to yield under the greatest diversity of climatic, edaphic and management conditions. They are grown under very different soil conditions, with varying managements, and are normally successively harvested throughout seasons and over years. Thus an appropriate dynamic representation of genotypic reaction to varying environments is of the utmost practical importance as well as of theoretical interest. 


\section{Materials}

The material considered here consists of five populations of cocksfoot, Dactylis glomerata, grown in two different locations over two years. The locations were chosen to provide differences in annual rainfall and seasonal temperatures. Harvests were taken successively five times in the first year following planting (frequent cuts) and twice in the second year (hay and aftermath cuts). Management differences are thus confounded with years. This is a typical procedure adopted at Aberystwyth for screening plant introductions; indeed, the material forms part of a programme designed to assess introduced and indigenous grasses under contrasting conditions, and I am indebted to my colleague, Mr Bruce Tyler, for these data.

The five populations included an indigenous cultivar (S.37), three natural populations introduced from Portugal and France (Bc 4651, Cantal and Nievre) and a hybrid between S.37 and $B c 4651$.

The experiment had three replicates per location with a single plot of ten spaced $(2 \mathrm{ft} . \times 2 \mathrm{ft}$.) plants per population in each replicate. The character studied was the weight in grams of fresh material harvested. The basic data used in analysis are the means of the ten plants in each plot.

\section{Analysis and Results}

\section{(a) Analysis of variance and phenotypic regressions}

Yields, averaged over replicates, are given in table 1 , together with a full analysis of variance. There is a vast range in individual harvest yields with a span of some 60-fold difference. Marginal means show that yields are affected by time, place and system of harvesting and that there are large differences between the populations. All these main effects are highly significant against the replicates error in the analysis of variance. But of most concern, the analysis shows that the populations interact significantly with all environmental effects, which can be presumed to include climatic, edaphic and management factors. Moreover, since all interactions are significant, no immediate generalisation can be made on the relative performance of these populations over even a restricted range of environmental contrasts; indeed, the analysis argues that valid comparisons can only be made in each environment separately.

Since the analysis of variance can give no further useful account of the genotype-environment interactions, we can now consider any dynamic relationship which exists between genotypic and environmental effects in the way proposed by Finlay and Wilkinson (loc. cit.). To this end each harvest is recognised as a different unspecified macro-environment which is measured quantitatively by the mean of all five populations (column means). For each variety the linear regression of individual values on these fourteen environmental means can now be computed. Following this, the sums of squares measuring the interactions of the populations with environments can be repartitioned into an item measuring differences between the slopes of the five regressions and a residual item which measures the scatter of points about the regression lines.

The results of this analysis are also given in table 1 , and it is immediately clear that the major part of the population $\times$ environment variance is explained by differences between the slopes of linear regressions. Thus the regression 


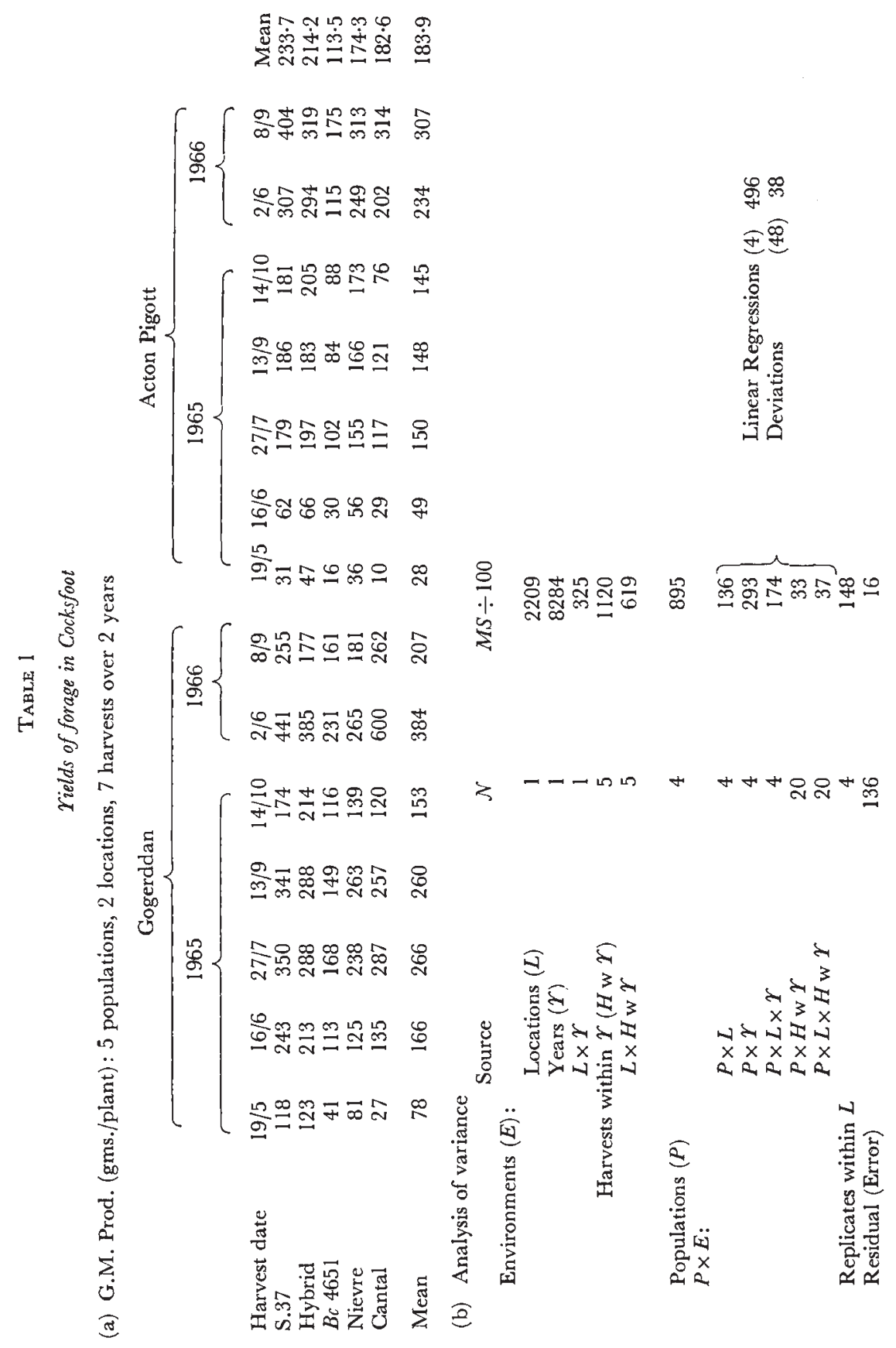


technique has transformed a complex tangle of genotype-environment interactions into an orderly series of linear, and hence predictable, responses. The small deviations mean square is still significantly greater than the replicates error item so that there are deviations from linearity which cannot be explained in terms of field error. These will be discussed later. In passing, it should be noted that this deviations item may be spuriously low because

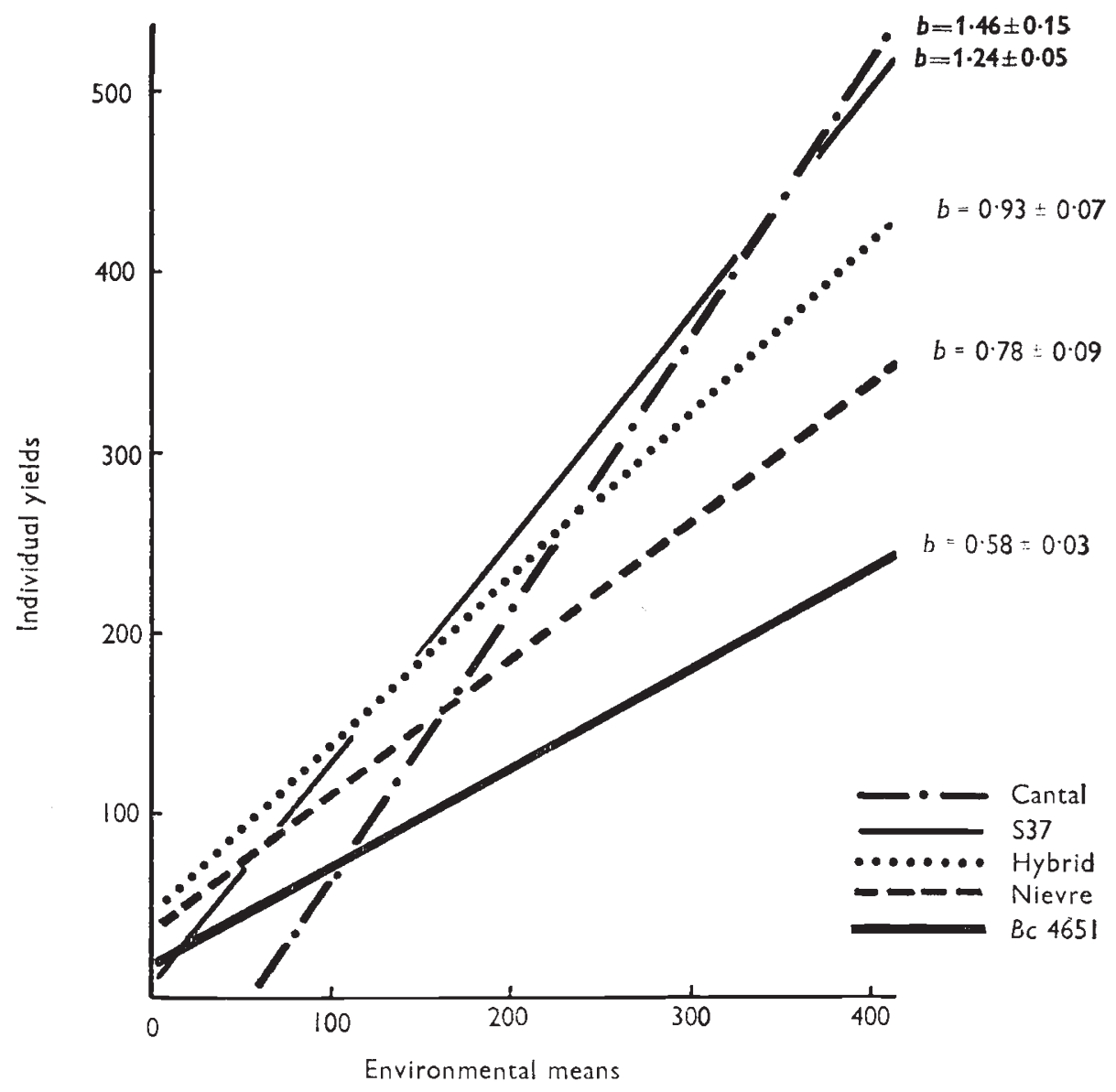

Low yielding High yielding

FIG. 1a.-Regressions of indiqidual population yields on mean yield for each harvest. All five populations.

measurements within locations are taken successively on the same plants and hence are not independent. Correlated errors which arise in this way, however, will be taken into account by the independent plantings of the replicates, and the replicates items in the analysis hav? sufficiently low values to indicate that they are not of major importance in the present study.

The actual regression lines with their coefficients and standard errors are shown in fig. la. To avoid confusion, individual points are not plotted, 
but two of the lines are reproduced in this detail in fig. $1 \mathrm{~b}$, and we shall discuss these first. They show that individual points are in strikingly good agreement with the fitted linear regressions as is reflected by the very low standard errors. For these two varieties, therefore, a remarkably accurate prediction of relative response is possible over this very wide range of environments.

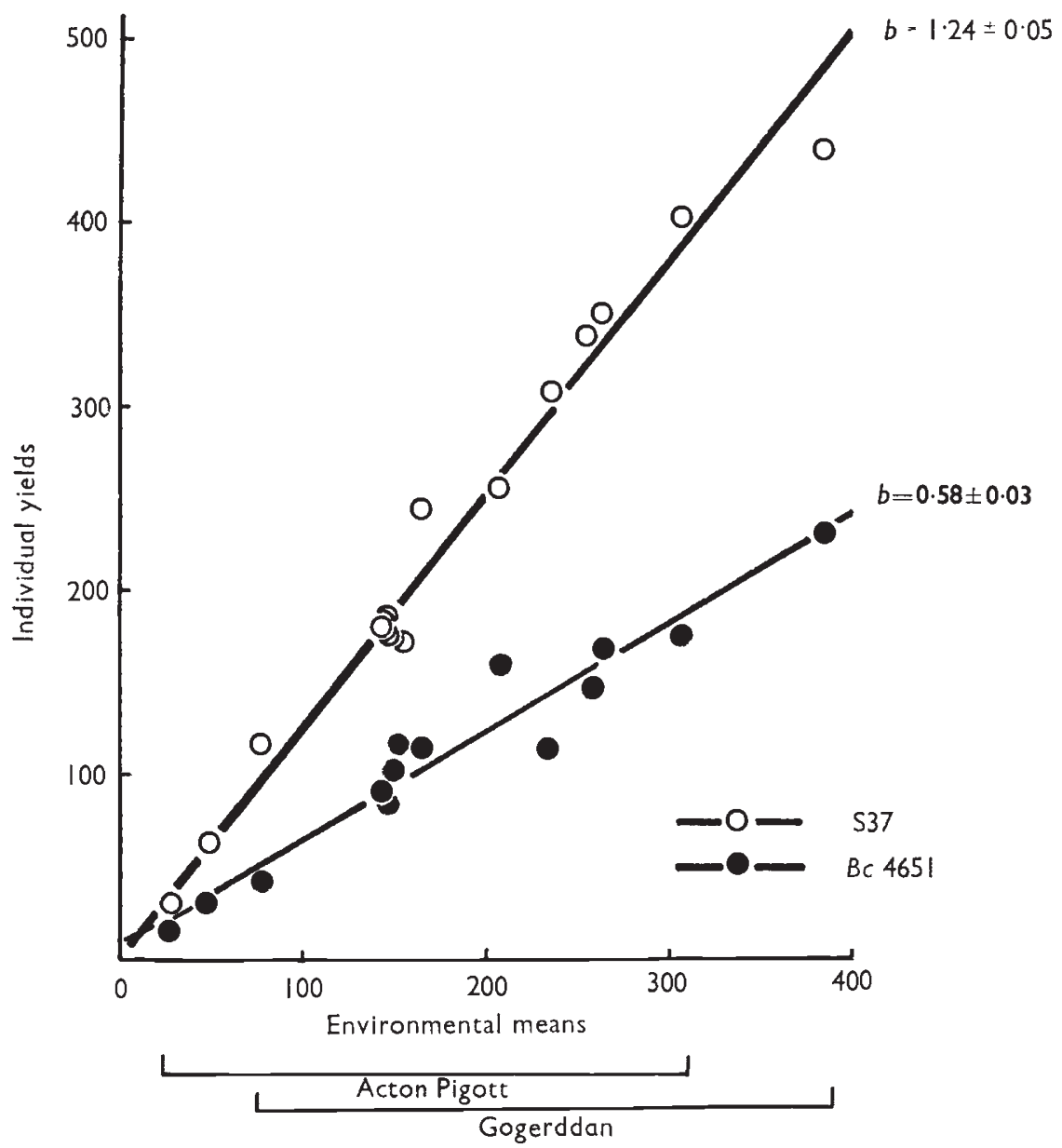

FIG. 1b.-Regressions of individual population yields on mean yield for each harvest. Two of the populations with individual points plotted.

To analyse as well as to emphasise this statement further, the ranges of expression achieved in each of the two locations (Gogerddan and Acton Pigott) are indicated by brackets along the horizontal axis of fig. $1 \mathrm{~b}$. It is evident that both mean and extreme expressions are different in the two locations. Yet the constancy of the linear regressions indicates that performance in one location could have been predicted from performance in the other. Obviously, the same conclusion may be drawn with regard to differences between seasons and managements. This strongly argues that if 
performance of genetic material is established relative to a standard in a comparatively restricted range of environments, the standard could be used as a reference for determining performance in other environments.

At this point it is of interest to recognise that environmental comparisons within locations were provided by successive harvests of regrowth from the same plants, whereas comparisons between locations were afforded by separate plantings. Evidently, in this material and for this character, vegetative regeneration and independent plantings from seed provide similar measures of response to environmental change.

The limits to useful extrapolation can, of course, only be determined by further experimentation. The present very divergent yields show that these limits can be wide, and fig. $1 \mathrm{~b}$ provides evidence that they can be even wider. Thus, if the lines are projected towards the origin, they intersect before the zero yield value is achieved. We might predict, therefore, that under conditions even more limiting on growth than were achieved in the present experiment, $B c 4651$ would outyield S.37. Such conditions are obtained in winters which are mild enough to allow some growth to take place but which is still restricted by low light intensities. This period was not covered in the present experiment, but it is a matter of observation and experimental record that under these conditions $B c 4651$ has in fact outyielded S.37. Indeed, this is the reason for our continued interest in $B c 4651$ and for its inclusion in the present material. It seems, therefore, that the regressions may be extrapolated and predictions made with a fair degree of accuracy over an extremely wide range of conditions.

Considering all five regressions lines (fig. 1a) it is apparent that similar predictive statements can be made about the relative performance of all these populations taken in any combination of two or more. Finlay and Wilkinson have developed these analyses so as to draw general inferences about adaptation in yielding ability among their cereal varieties. In doing so they have used both the variety means and regression values jointly in classifying varieties according to general or specific adaptability to different environments. Since results so far show that regressions are linear over a wide range of environments, estimates of the regression coefficients should be unaffected for a given set of populations even if the sample of environments is biased. The distribution of population mean values, on the other hand, depends directly on the specific set of environments considered. The former statistics, therefore, are the critical determinants in deciding on relative adaptabilities, and the means serve only to discriminate between regression coefficients of equal value or to specify performance within a limited set of environments.

The regression coefficients are in effect measures of responses to increments in an improving environment. Since these increments are measured by the mean of all populations, then the average response for any set of populations under consideration must have a regression coefficient of 1.0 . The cultivar S.37 had an above average response $(b=1.24)$ and was a consistently high yielder in all above-average environments. This general adaptation to a fairly wide range of good environments is to be expected from a variety deliberately bred for high yield during the main growing season and under fertile conditions. $B c 4651$, on the other hand, has a response well below the average $(b=0.58)$ and is adapted to low-yielding environments, while by contrast Cantal is adapted only to the highest yielding 
environments, and is marked by a high response $(b=1.46)$, but a comparatively low mean yield in this range of environments. These results could be used as a basis for considering more specifically the behaviour of each population in relation to its origin and thus more critically to examine the importance of limiting environmental factors. Such aspects will, however, be discussed in detail elsewhere, since we are here concerned only with the general applicability of the techniques.

\section{(b) Stability}

The standard errors attached to the regression coefficients in fig. la have been calculated separately for each linear regression from deviations within the appropriate set of 14 points. They are very variable and reflect the fact that mean squares measuring the scatter of points about individual regression lines are not homogenous $\left(\chi^{2}=21.0\right.$ and $\mathrm{P}<0.001$ on Bartletts test). Thus the joint mean square measuring the overall deviations from regressions, presented for convenience in table $1 \mathrm{~b}$, is not strictly valid. Indeed, there can be little doubt that the extent of the deviations from regression is specific to, and hence characteristic of, particular populations. It must be emphasised that in no case did the graphs indicate any relationship other than linear, individual points being scattered at random about the fitted straight line. Standard errors measuring this scatter may thus be taken as measures of the "stability of response" exhibited by each population.

It is now apparent that phenotypic expression ( $Y$ ) of a particular population $(i)$ in a specific environment $(j)$ depends on three genotypic properties: a mean expression, a linear response to environment and residual deviations from regression. These parameters are exactly those proposed by Eberhart and Russell (1966) to define their "model for stability" which they express as

$$
\mathrm{Y}_{i j}=\mu_{i}+\beta_{i} I_{j}+\delta_{i j}
$$

where $\mu_{i}$ is the mean of $i$ th variety over all environments, $\beta_{i}$ is the regression coefficient that measures the response of the $i$ th variety to varying environments against the environmental index $I_{j}$, which is obtained as the mean of all varieties in the $i$ th environment, and $\delta_{i j}$ is the deviation from regression of the $i$ th variety in the $j$ th environment.

Since the linear regressions represent very definite and measureable responses to the environment, it is no longer profitable to consider this component of genotype-environment interactions as a measure of stability in the way described by Finlay and Wilkinson. The term " stability" should now rather be reserved to describe measurements of unpredictable irregularities in the response to environment as provided by the deviations from regression. This distinction has also been proposed by Eberhart and Russell (loc. cit.). It is worth consideration also that such a concept of stability in response to changing environments conforms very closely to the concept of " developmental homeostasis" as discussed by Lerner (1958).

Reference to fig. la shows that on these premises cultivar S.37 and population $B c 4651$ are most stable in response, the hybrid between them less so, and the two populations Cantal and Nievre the least stable. Further statistical evidence on these points is presented in a later section. 


\section{(c) Genetic parameters}

The response regressions, as discussed so far, have provided dynamic representations of genotypes which allow prediction over environments. Obviously we require to know to what extent and in what way this additional characterisation of the genotype is inherited.

Methods of describing and measuring components of genetic variation (Mather, 1949) have been extended by Mather and Morley Jones (1958) to include parameters specifying environmental effects and the interaction of genotype with environment. These have been adapted by Bucio Alanis (1966) to the analysis of differences in height between two inbred lines of $\mathcal{N}$ icotiana rustica grown in a number of seasons and locations. Variation in the character was partitioned into a constant genetic difference $[d]\left(=\frac{1}{2}\right.$ the mean parental difference), an environmental effect $\epsilon$ which is measured by the mid-parental value in each environment, and the interaction of genotype and environment $\gamma$ which is the deviation of the parental difference from $[d]$ in each environment. All values are expressed as deviations from the overall mid-parent $(\mu)$ so that phenotypic values in a particular environment may be written in the form

$$
\begin{aligned}
& P_{1}=\mu+[d]+\epsilon+\gamma d \\
& P_{2}=\mu-[d]+\epsilon-\gamma d
\end{aligned}
$$

It was found that the genotype-environment interaction $\gamma$ was a linear function of the effect of the environment $\epsilon$. Thus logical analysis of character variability into components for genetic, environmental and their joint effects has produced exactly the same measure of the environment as that used by Finlay and Wilkinson on a priori grounds, and has also shown the same linear relationship between this and genotypic responses.

Bucio Alanis and Hill (loc. cit.) have extended the analysis to include the $F_{1}$. The phenotypic value for this generation in any specific environment is given by the expression:

$$
F_{1}=\mu+[h]+\epsilon+\gamma h
$$

where $\mu$ is the grand mean for parents, $[h]$ is the mean deviation of the $F_{1}$ from $\mu$, and $\gamma_{h}$ is the deviation from [h] in that environment. They found that $\gamma_{h}$ was also a linear function of $\epsilon$ but a different function from $\gamma_{d}$. Thus the response to the environment of the heterozygotic component $[h]$ is not the same as that of the homozygotic component [d].

This form of analysis can be applied to the two populations, S.37 and $B c 4651$, and the hybrid between them, which are included in the present experiment. The populations are not inbred and so cannot be regarded as homozygous; however, they are sufficiently divergent to be classified as sub-species and it may therefore be considered that the genes controlling differences between them are largely in the homozygotic state. Consequently these differences may be analysed in the same way as differences between inbred lines, the residual heterozygosity being important only in so far as it inflates the error of estimation. Further particulars of these two populations and the hybrid can at this stage be briefly considered. S.37 in common with the majority of cocksfoot cultivars, is a natural tetraploid $(2 n=28)$ of the sub-species glomerata. $B c 4651$, on the other hand, is an introduced Portuguese diploid $(2 n=14)$ of the sub-species lusitanica. The 
hybrid was produced at this station by Dr Borrill who crossed the autotetraploid of $B c 4651$ with S.37, and this hybrid has turned out to be remarkably stable. So far as can be seen there is little preferential pairing within genomes and thus we may presume tetrasomic segregational patterns. The hybrid material used was second generation so that the fully heterozygous, duplex condition will not have been maintained and heterozygotic effects such as dominance and certain non-allelic interactions may be underestimated. In the present study however, absolute values for these quantities are not at issue, but rather their change with environment and for this reason the hybrid is regarded as an $F_{1}$. For the same reason we need not concern ourselves with the special properties of $[d]$ and $[h]$ values when estimated from tetraploid material.

\section{TABLE 2}

Estimated values of genetic and environmental parameters for $S .37\left(\mathrm{P}_{1}\right)$ and $\mathrm{Bc} 4{ }_{45} \mathrm{I}\left(\mathrm{P}_{2}\right)$ and their hybrid $\left(=\mathrm{F}_{1}\right)$

\begin{tabular}{|c|c|c|c|c|}
\hline Environment & $=\frac{1}{2}\left(P_{1}+P_{2}\right)-\mu$ & $\begin{array}{c}{[d]+\gamma_{d}} \\
=\frac{1}{2}\left(P_{1}-P_{2}\right)-\mu\end{array}$ & $\begin{array}{l}{[h]+\gamma_{h}} \\
=F_{1}-\mu\end{array}$ & $\begin{array}{l}\text { Potence ratio: } \\
{[h]+\gamma_{h} /[a]+\gamma_{d}}\end{array}$ \\
\hline 1 & $-94 \cdot 5$ & 38.5 & $43 \cdot 5$ & $1 \cdot 13$ \\
\hline 2 & $4 \cdot 0$ & $65 \cdot 0$ & $35 \cdot 0$ & 0.54 \\
\hline 3 & $85 \cdot 0$ & $91 \cdot 0$ & $29 \cdot 0$ & $0 \cdot 32$ \\
\hline 4 & $71 \cdot 0$ & $96 \cdot 0$ & $43 \cdot 0$ & 0.45 \\
\hline 5 & $-29 \cdot 0$ & $29 \cdot 0$ & $69 \cdot 0$ & $0 \cdot 24$ \\
\hline 6 & $162 \cdot 0$ & $105 \cdot 0$ & $49 \cdot 0$ & 0.47 \\
\hline 7 & 34.0 & $47 \cdot 0$ & $-31 \cdot 0$ & -0.66 \\
\hline 8 & -150.5 & $7 \cdot 5$ & $23 \cdot 5$ & $3 \cdot 13$ \\
\hline 9 & $-128 \cdot 0$ & $16 \cdot 0$ & $20 \cdot 0$ & $1 \cdot 25$ \\
\hline 10 & -33.5 & 38.5 & $56 \cdot 5$ & 1.47 \\
\hline 11 & $-39 \cdot 0$ & $51 \cdot 0$ & $48 \cdot 0$ & 0.94 \\
\hline 12 & $-39 \cdot 5$ & 46.5 & $70 \cdot 5$ & 1.52 \\
\hline 13 & $37 \cdot 0$ & $96 \cdot 0$ & $83 \cdot 0$ & 0.86 \\
\hline 14 & $115 \cdot 5$ & 114.5 & $29 \cdot 5$ & $0 \cdot 26$ \\
\hline $\begin{array}{c}\text { Mean of } \\
\text { Environments }\end{array}$ & $\begin{aligned} \mu & =174 \\
& =\frac{1}{2}\left(\widetilde{P}_{1}+\bar{P}_{2}\right)\end{aligned}$ & $\begin{aligned} {[d] } & =60 \cdot 1 \\
& =\frac{1}{2}\left(\widetilde{P}_{1}-\widetilde{P}_{2}\right)\end{aligned}$ & $\begin{aligned} {[h] } & =40 \cdot 2 \\
& =F_{1}-\mu\end{aligned}$ & {$[h] /[d]=0.67$} \\
\hline
\end{tabular}

Table 2 gives estimates of the parameters $\epsilon,[d]+\gamma_{d}$ and $[h]+\gamma_{h}$ computed from values in table 1 a for all 14 environments taken in order. Values of $\mu,[d]$ and $[h]$ are also given together with the method of estimation. The relationship between $\epsilon$ and $\gamma_{d}$ and $\gamma_{h}$ can be found from the scatter diagrams of fig. 2, in which $[d]+\gamma_{d}$ and $[h]+\gamma_{h}$ respectively have been plotted against $\epsilon ;[d]$ and $[h]$ are constants. As expected from the previous phenotypic graphs, $\gamma_{d}$ is linearly related to $\epsilon$ and has a regression coefficient $\left(\beta_{d}\right)$ of $0.35 \pm 0.03$ which may now be used as a measure of the function of the effect of the environment on $[d]$.

In marked contrast, $[h]+\gamma_{h}$ does not appear to be a function of the environment $\left(\beta_{h}=0.04 \pm \mathrm{p} \cdot 08\right)$, and thus within the limits of experimental error $[h]$ can be regarded as a constant regardless of the environment. This quantity $[h]$ is an estimate of potence and contains effects due to dominance and non-allelic interaction, and is influenced by the distribution of genes in the parents. Although $[h]$ appears to be constant, because $[d]$ varies with environment, the potence ratio (table 2) varies from 3.13 in a poor environment to 0.24 in a good environment. In general terms, therefore, expression varies from apparent overdominance to only partial dominance. 


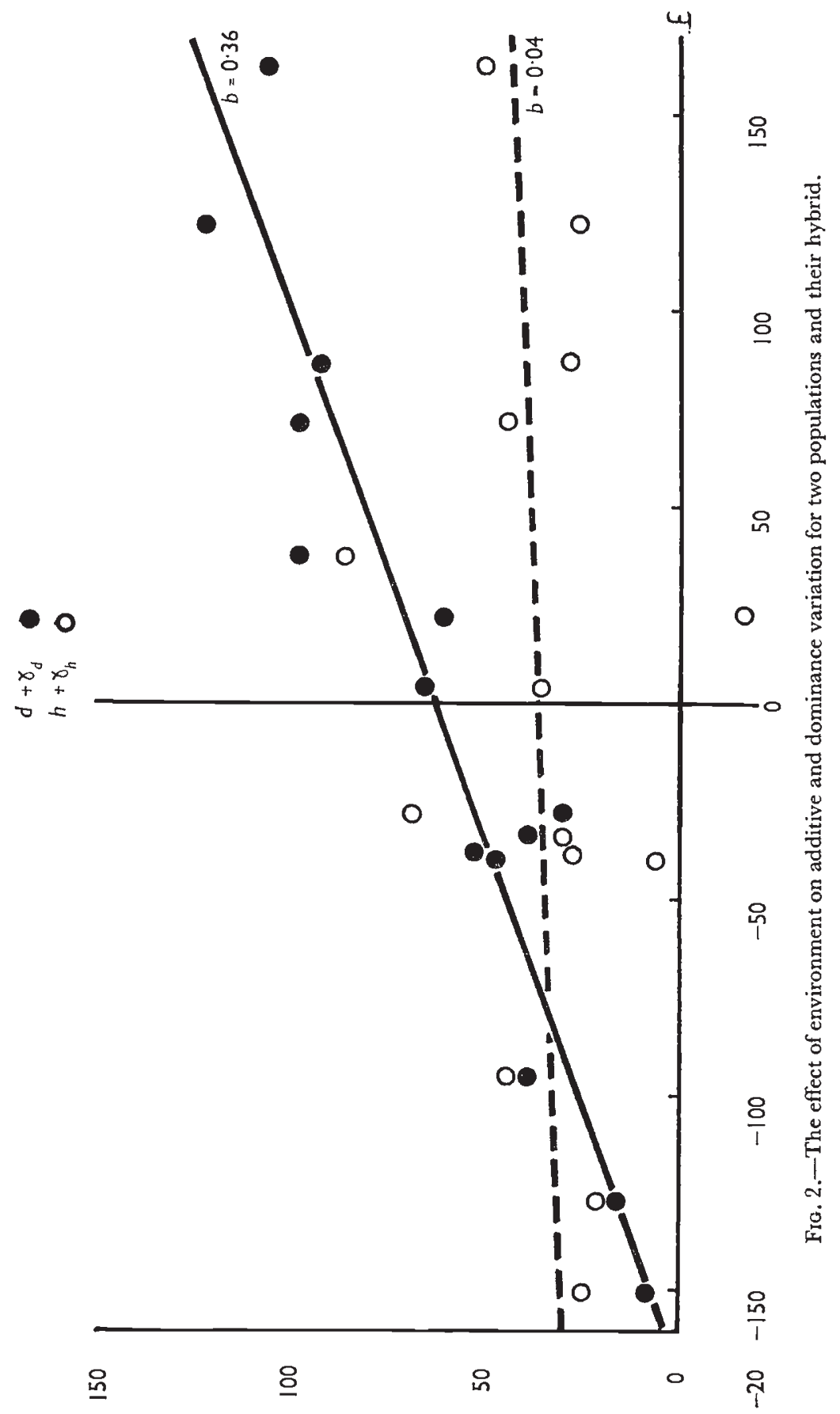


These parameters can be used to construct the phenotypic regressions for the parents and the hybrid. Thus in the $j$ th environment,

$$
\begin{aligned}
& P_{1 j}=\mu+[d]+\left(1+\beta_{d}\right) \epsilon_{j} \\
& P_{2 j}=\mu-[d]-\left(1+\beta_{d}\right) \epsilon_{j} \\
& F_{1 j}=\mu+[h]+\left(1+\beta_{h}\right) \epsilon_{i}
\end{aligned}
$$

These are the phenotypic expressions given earlier, but with $\gamma_{d}$ and $\gamma_{h}$ expressed as functions of the environment. Substituting estimated values we get the regression lines shown in fig. 3. Alternatively we could construct the

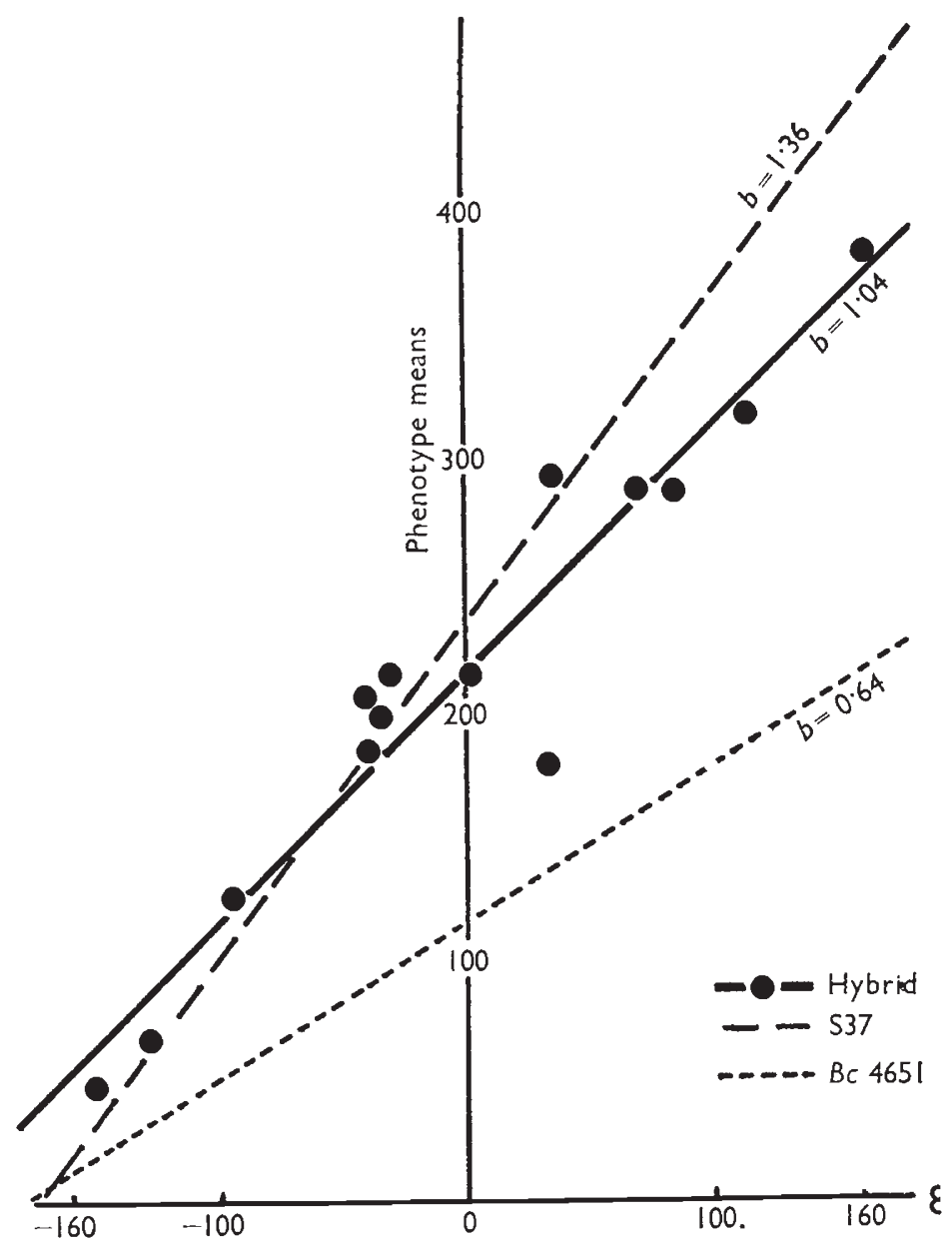

FIG. 3.-Phenotypic regressions for parental populations and hybrid.

regressions from the observed phenotypic values as in the previous section. Indeed, the only difference between these regressions and the corresponding ones in fig. la is that in this instance the measure of the environment $(\epsilon)$ is provided by the two parental varieties only and is expressed as a deviation from the overall mean. This alters the absolute slopes of the regressions, but in no way affects their behaviour relative to each other. As an indication of 
scatter, observed values for the hybrid are plotted and may be compared with those for the parents in fig. $1 \mathrm{~b}$.

These phenotypic regression lines (fig. 3) usefully translate the genetic parameters into phenotypic expression. We see that with $\beta_{h}=0$ the response of the hybrid to improving environments is exactly intermediate to that of the two parents. Because of the constant $[h]$ value, however, the position of the slope is such that the expression varies from heterosis in below average environments to intermediacy in above average environments. In plant breeding terms, a phenomenon which could be recognised as a feature of special combining ability in one environment becomes transmuted to an expression of general combining ability in another environment. And yet it requires no more complicated explanation than partial dominance for mean expression and additivity for a linear response to environmental change.

\section{(d) Heritabilities}

The term " heritability" is variously defined according to its usage (see Sprague, 1966). In its simplest form it is "the portion of the observed variance for which difference in heredity is responsible" (Lush, 1949). This is termed broad-sense heritability and can be expressed as $H_{B}=\frac{G}{G+E}$ where $G$ is the total genetic variance and $E$ is the environmental variance. It requires only that adequate measures of environmental effects are obtained and so can be estimated from the replication of an appropriate group of genetic entities grown under one or more sets of environmental conditions. It should be noted that genotype-environment interaction variances are implicitly contained in the denominator. As a guide to progress expected from selection this estimate is, of course, inadequate since the total genetic variance includes dominance and epistatic dominance components which are not retained by selection. For this purpose, a more useful estimate has been proposed which includes only additive components (here designated $D$, following Mather's notation) in the numerator. This is termed narrow-sense heritability and is expressed as $H_{N}=\frac{D}{G+E}$. Its estimation requires breeding tests designed to measure $D$.

Both estimates depend on gene frequencies and gene distributions in the material under test, and on the environmental conditions under which it is grown. Heritabilities determined for different populations under different levels of environmental control are not therefore directly comparable. Under defined circumstances they do, however, form useful standards for comparing the degree of genotypic $\left(H_{B}\right)$ or genetic $\left(H_{N}\right)$ control of different attributes. It is in this context that they are considered here in order to emphasise the increased information obtained in characterising the genotype by its response to the environment as well as by the mean (i.e. according to the linear regression term $y=a+b_{x}$ ).

Considering again only those genes by which the populations differ, estimates of both kinds of heritability can be obtained from the present data. To this end, component analyses of variation are proposed in table $3 \mathrm{~b}$, assuming a mixed model (Snedecor, 1956) with a fixed number of populations and a random sample of environments. This is admissible if we are concerned only with the effect of the environment on the genetic differences 
between a specific number of lines, which is the case here. On the other hand, if we were concerned with an adequate measure of the environment per se, we should obviously have to consider a random sample of populations since, as the actual analysis of the data (table $3 a$ ) shows, such estimates of the environments are affected by the choice of genotypes and thus contain components due to genotype-environment interactions. The components of variation in table $3 \mathrm{~b}$ are presented in two forms according to whether or not the item for interaction is partitioned into mean squares measuring differences between linear regression and the residual deviations from these.

TABLE 3

Component Analyses

(a) Separate analyses of variance for paired populations and the hybrid

\begin{tabular}{lrccc}
\multicolumn{1}{c}{ Source } & $\mathcal{N}$ & $\begin{array}{c}\text { Cantal and } \\
\text { Nievre M.S. }\end{array}$ & $\begin{array}{c}\text { S.37 and } B c 4561 \\
\text { M.S. }\end{array}$ & $\begin{array}{c}\text { Hybrid } \\
\text { M.S. }\end{array}$ \\
Populations $(G)$ & 1 & 15 & $3073 * * *$ & - \\
Environments $(E)$ & 13 & $783 * * *$ & $502 * * *$ & $3561 * * *$ \\
$G \times E$ : & 13 & $150 * * *$ & $75 * * *$ & - \\
$\quad\left\{\begin{array}{l}\text { Regression }(b) \\
\text { Deviation }(d)\end{array}\right.$ & 1 & $1141 * * *$ & $828 * * *$ & $3255^{* * *}$ \\
Replicates (in locations) & 12 & $80 * * *$ & 12 & $25 *$ \\
Error & 52 & 14 & 65 & 244 \\
& & 4 & 14 & $12[24] \dagger$
\end{tabular}

$* * *$ and $* P<0.001$ and $<0.05$ respectively against appropriate errors as indicated below. $\dagger$ Value of $\mathcal{N}$ corrected for hybrid.

(b) Components assuming a fixed number $(\mathrm{g})$ of population and a random sample (e) of environments with $\mathrm{n}$ replications (Mixed Model)

Source

Populations $(G)$

Environments $(E)$

$G \times E$ :

Regression (b)

Deviation $(d)$

Error
Parameters estimated

$\begin{array}{ll}\text { Genotype measured by } & \text { Genotype measured by * } \\ \overline{\mathrm{y}} & \bar{y}+\beta \epsilon \\ \sigma^{2}+n \sigma_{G E^{2}}+n e \sigma_{G}{ }^{2} & \sigma^{2}+n \sigma_{d}{ }^{2}+n e \sigma_{G}{ }^{2} \\ \sigma^{2}+n g \sigma_{E}{ }^{2} & \sigma^{2}+n g \sigma_{E}{ }^{2} \\ \sigma^{2}+n \sigma_{G E}{ }^{2} & \\ & \sigma^{2}+n \sigma_{d}{ }^{2}+n e \sigma_{b}{ }^{2} \\ & \sigma^{2}+n \sigma_{d}{ }^{2} \\ \sigma^{2} & \sigma^{2}\end{array}$

$* y=$ population mean, $\beta e=$ linear regression on environmental measure.

The corresponding variance analysis presented in table 3 a subdivides the data as shown. S.37 and $B c 4651$ are treated as parental lines and their mean value also provides the environmental measure for the individual analysis of the hybrid data. For contrast Cantal and $\mathcal{N i e v r e}$ are analysed as a separate pair. These analyses provide further evidence on differences between the populations with regard to the stability of response as measured by the regressions. Thus in the case of $\mathrm{S} .37$ and $B c 465 \mathrm{l}$ all interactions with the 14 macro-environments are explained by differences between linear regressions, the deviation mean square being of the same order as the error mean square measuring fluctuations in the uncontrolled micro-environment (measured by replicates). These lines may be regarded therefore as highly stable in response; we may perhaps say that they display a high degree of 
developmental homeostasis. Interestingly, the hybrid between them shows significant deviations from regression suggesting that the newly derived material from this very wide cross has not achieved the developmental stability of either parent. The other pair of populations, Cantal and Nievre, show marked deviations from linear regression and thus appear relatively unstable in response. Nevertheless, it can again be clearly seen for all populations that by far the greatest portion of genotype-environment interactions is explained by linear response.

\section{TABLE 4}

Broad-sense $\left(\mathrm{H}_{\mathrm{B}}\right)$ and narrow-sense $\left(\mathrm{H}_{\mathrm{N}}\right)$ heritabilities

Over environments Genotypes characterised by

Estimate and source

$H_{B}=\frac{G}{G+G E+E} *$

All populations

Pairs of populations:

(a) Nievre; Cantal

(b) S.37; Bc 4651
Within environments

$0 \cdot 51-0 \cdot 89$

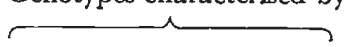

$\bar{y}$

$0 \cdot 37$

Nil

0.67

0.52 $\bar{y}+\beta \epsilon$

0.59

0.46

$0 \cdot 87$
$0 \cdot 67$

$H_{N}=\frac{D}{G+G E+E} *$ :

$\mathrm{S} .37 \times B c 4651$

$0 \cdot 03-0 \cdot 86$

* $H_{B}$ computed from table 3 :

for $\bar{y}=\frac{\sigma_{G}^{2}}{\sigma_{G}^{2}+\sigma_{G E}^{2}+\sigma_{2}} ; \quad$ for $\bar{y}+\beta \epsilon=\frac{\sigma_{G}^{2}+\sigma_{b}^{2}}{\sigma_{G}^{2}+\sigma_{b}^{2}+\sigma_{s}^{2}+\sigma^{2}}$

* $H_{N}$ estimated from components and table 3 :

$$
\begin{aligned}
\text { for } \bar{y} & =\frac{\sigma_{[d]}^{2}}{\sigma_{[d]}^{2}+\sigma_{[h]}^{2}+\sigma_{G E}+\sigma^{2}} \\
\text { for } \bar{y}+\beta \epsilon & =\frac{\sigma_{[d]}^{2}+\sigma^{2} \beta d}{\sigma_{[d]}^{2}+\sigma_{[h]}^{2}+\sigma^{2} \beta d+\sigma^{2} \beta h+\sigma_{s}^{2}+\sigma^{2}}
\end{aligned}
$$

where $\sigma_{[d]}^{2}=[d]^{2}$ corrected for environmental effects and put on a single plot basis $\left(=\sigma_{G}^{2}\right.$ by the assumptions made).

and $\sigma_{[h]}^{2}=[h]^{2}$ corrected for environmental effects and put on a single plot basis.

It must be noted that these analyses indicate that estimates of microenvironmental (replicate) variation are not homogenous among the populations. This need not concern us at this juncture, however, since mean and variance are not obviously correlated and so no simple rescaling of the data is indicated.

Estimates of the components of variation can be obtained from the analysis by the use of the formulae in table $3 \mathrm{~b}$. Similar components can be obtained for all five populations taken together from the appropriate modification of the analysis presented in table $1 \mathrm{~b}$. In addition, components of genetic variation can be obtained from analysis of data within environments. Intra-class correlations can then be made to yield estimates of broad-sense heritabilities (i.e. the proportion of genotypic to total variation) and some of these are given in table 4. 
Taking all five populations together and for environments separately, heritabilities range from 0.5 in poor environments, where expression is limited, to 0.89 in the best environment; thus emphasising the obvious fact that genetic differences are generally best measured in non-limiting environments. If we still characterise the populations by mean expression, but take all environments into account, heritability drops to 0.37 because, as the graphs show, the order of performance is changing. But if the populations are characterised by mean and response (i.e. $P=\bar{y}+\beta \epsilon)$, then heritability, or that portion of the total variation which can be recognised as genotypic, increases to almost 60 per cent.

Taking the populations two at a time, the difference between Cantal and Nievre has a heritability of zero if classified on mean expression which suggests that there are no genetic differences between them, whereas the second method of calculation gives a heritability of 46 per cent. This is because they have similar means but different responses, giving the classical type of extreme interaction wherein their order of performance is completely reversed and more or less counterbalanced from below average to aboveaverage conditions. That this heritability value is still comparatively low reflects the instability in their response noted earlier. A less dramatic improvement in heritability is achieved by considering response as well as mean in the case of S.37 and $B c 4651(0.67$ to $0 \cdot 87)$, for which the order of performance does not change over the range of environments sampled.

If we assume that all genes by which S.37 and $B c 4651$ differ are in the associated phase, and that dominance and interaction are uni-directional, then narrow-sense heritability ( $=$ the proportion of additive to total variation) can be calculated from a joint analysis of parent and hybrid data, using the components from table 2 as indicated in table 4 . Within environments it varies from negligible to fairly high as foreshadowed by the presence or absence of heterosis. Over environments, taking into account linear response again effects a significant improvement in the heritability value. Very clearly the potential value of this combined measurement as a selection criterion is considerable.

\section{Discussion}

The results presented here well illustrate that the regression analyses proposed separately by Finlay and Wilkinson (loc. cit.) and by Bucio Alanis and Hill (loc. cit.) are powerful tools in the analysis of genotype-environment interactions. The essence of the method is that appropriate biological material can be used to quantify environments and so provide a basis for measuring genotypic response to changing conditions. So far, the measure of the environment has been provided by the mean value of the set of genotypes under test, and the response of each individual genotype then determined by the regression of its individual values on these means for a range of environments. For the five grass populations considered here the yield responses so measured were linear, and differences between the populations could be largely explained by differences between the slopes of their linear regressions. The regressions provided a means of accurately predicting relative performance over a wide range of environments, and could also be used to simplify varying relationships between parents and offspring under changing conditions. 
The technique has been used to analyse yield in a number of other experiments involving different grass populations and species from those reported here. In all cases where fair comparisons could be made between the populations, linear relationships have been established which provide useful measures of the dynamic relationships between genotype and environment. Thus in one guise or another the technique has now been successfully applied to a wide range of plant material under diverse environmental conditions. In each case the regressions have been linear. This was so for the statistical " artifice" used by Yates and Cochran (1938) to reveal relationships between general soil fertility and varietal differences in cereal yields, for the " adaptation measurements" of Finlay and Wilkinson (1963) with regard to cereal yield in Australia, as well as here in considering yields of forage in grasses under extreme ranges of climatic, edaphic and management conditions. It was also so in the sophisticated genetical studies on final height in Nicotiana rustica carried out at Birmingham (Bucio Alanis et al., 1966; Perkins and Jinks, 1968) where, most significantly, the linear functions can be shown to have predictive value over generations as well as environments (Bucio Alanis, personal communication).

Until now hopes that genotype-environment interactions could be successfully accommodated in the handling of continuous variation have not been optimistic. The position was well summarised by Sprague (1966) who states: "Genotype-environemnt interactions constitute an important limiting factor in the estimation of variance components and in the efficiency of selection programmes. Unfortunately we know little concerning the environmental factors which contribute to such interactions. Even if such information were available, the possibility of materially reducing such interactions under field conditions appears somewhat questionable". Perhaps this attitude, that we should seek to minimise such interactions, has for too long stultified our approach to the problem. We now see that measured against an appropriate scale, the combined effects of genotype and environment do not behave in the disorderly, unpredictable way conveyed by the term " interaction", but can be portrayed as orderly and predictable responses of the genotype to a regulating medium. This lends hope that changes in estimated genetic variances and components from location to location, and from year to year, can be explained by simply expressed and simply inherited linear responses.

Hitherto, the stumbling block has been obtaining an appropriate scale against which to measure genotypic response, perhaps because we have been over-concerned to use physical measurements of individual environmental factors. Yet a logical development of the genetical axiom that the phenotype is a product of the genotype and its environment leads to the acceptance that it is just as apposite to grade an environment according to the mean expression of a range of genotypes, as it is to quantify a genotype by its average expression over a range of environments. For quantitative studies the fact that these measurements do not specifically describe the variable factors of the environment need not deter us any more than the fact that genotypic measurements do not specify the underlying biochemical processes. Indeed, this joint measure should ultimately provide a basis for better understanding physical limits in the environment as well as physiological control by the genotype.

Of course, that environmental effects are reflected in biological measurement is implicit in all analyses of variance of biological material. It has only 
now become profitable to recognise that such measurements can characterise the environment as well as the genotype with the discovery that, when measured on this common scale, the response of individual genotypes can be expressed as simple linear functions of the environmental measures to which they collectively subscribe. Certainly we need to know more of the limits between which these linear relationships hold, or can be expected to hold, both by experimental investigation and from mathematical models. But, if early promise is fulfilled, we may expect that appropriate measurements will provide linear response functions which will be invaluable in selection programmes and may also contribute to a better understanding of quantitative gene action. Whether these can easily be achieved will obviously depend on practical considerations. Not the least of these will be a modified approach to experimental design, leading to better measurement over maximised environmental differences, and perhaps having less concern with the accurate determination of micro-environmental fluctuations.

\section{Summary}

1. Since the phenotype is the product of the genotype and its environment, it is just as apposite to numerically grade an environment according to the mean expression of a range of genotypes as it is to quantify a genotype by its average expression over a range of environments.

2. Published results by different authors have shown for yield in cereals and for height in Nicotiana rustica that, measured on this common scale, the performances of individual genotypes are linear functions of the environmental values to which they collectively subscribe.

3. Thus the linear regressions of individual genotypic values on the mean value of all genotypes for each of a number of environments provide measures of response which can be used to predict relative performance over a range of environmental conditions.

4. This paper describes the application of this technique to the analysis of forage yield in perennial grasses, where successive harvests are subject to marked contrasts in the external environment.

5 . The wide range of genetic material studied showed marked interactions with contrasting climatic, edaphic and management conditions. The major part of these could be explained by differences between linear responses as estimated by the regressions.

6. The results have been used to demonstrate that the method can be a powerful means of predicting relative performances of populations and their hybrids over seasons, years and locations.

7. The evidence supports the view that this technique may constitute a major advance in the genetic study of quantitative characters. The implications of this are briefly discussed.

Acknowledgments.-I am indebted to Professor J. L. Jinks for focussing my attention on recent developments in the analysis of genotype-environment interactions at Birmingham, and for helpful advice. I gratefully acknowledge the generosity of Mr B. F. Tyler in allowing me to use data from his trials with introduced plant material, and of Dr M. Borrill for permission to use unpublished data on a recently produced species-hybrid in Dactylis. I am also grateful to Dr J. Hill for valuable discussion and to the Director of the Welsh Plant Breeding Station, Professor P. T. Thomas, for his unflagging interest. 


\section{REFERENCES}

BUCio ALANis, L. 1966. Environmental and genotype-environmental components of variability. I. Inbred lines. Heredity, 21, 387-397.

BUCIO ALANIS, L., AND HILL, J. 1966. Environmental and genotype-environmental components of variability. II. Heterozygotes. Heredity, 21, 399-405.

EBERHART, s. A., AND RUSSEll, w. A. 1966. Stability paramters for comparing varieties. Crop Sci., 6, 36-40.

FINLAY, K. W., AND WILKINson, G. N. 1963. The analysis of adaptation in a plant breeding programme. Aust. F. Agric. Res., 14, 742-754.

JINKs, J. L., AND STEVENS, J. M. 1959. The components of variation among family means in diallel crosses. Genetics, 44, 297-308.

LERner, I. M. 1958. The Gentic Basis of Selection. John Wiley and Sons, Inc., New York. Lush, J. L. 1949. Heritability of quantitative characters in farm animals. Proc. 8th Int. Congr. of Genetics Suppl. Vol. Hereditas, 356-375.

MATHER, K. 1949. Biometrical Genetics. Methuen, London.

MATHER, K., AND JONES, R. MORLEY. 1958. Interaction of genotype and environment in continuous variation. I. Description. Biometrics, 14, 343-359.

PERKINS, JEAN, AND JINKs, J. L. 1968. Environment and genotype-environmental components of varability. III. Multiple lines and crosses. Heredity, 23, in press.

SNEDECOR, G. W. 1956. Statistical Methods. Iowa State University Press, Ames, Iowa, U.S.A.

SPRAGUE, G. F. 1966. Quantitative genetıcs in plant improvement. Plant Breeding-A Symposium Held at Iowa State University, pp. 315-354. Ed. K. J. Frey. Iowa State University Press, Ames, Iowa.

yates, F., AND COChRan, w. G. 1938. The analysis of groups of experiments. F. Agric. Sci. Camb., 28, 556-580. 